\title{
Bienestar Subjetivo en Maximizadores y Satisfacedores
}

\author{
Subjective Well Being in Maximizers and Satisfecers
}

\author{
Emilio Moyano-Díaz \\ Felipe Cornejo \\ Marcela Carreño \\ Alejandra Muñoz \\ Universidad de Talca, Chile.
}

(Rec: 26 de junio 2012 / Acept: 05 de septiembre 2013)

\section{Resumen}

El propósito aquí es evaluar una tendencia decisional cuyos polos son la simple satisfacción hasta la maximización, y sus efectos eventuales sobre el bienestar subjetivo (B.S.), en sus componentes afectivo (felicidad) y cognitivo (satisfacción vital). Una muestra de 209 estudiantes de la Facultad de Psicología de la Universidad de Talca, respondió un cuestionario con cuatro instrumentos para medir maximización-satisfacción y B.S. Los resultados muestran diferencias significativas entre los participantes para el componente cognitivo -satisfacción vital- del B.S., donde quienes son satisfacedores tienen una mejor valoración de sus vidas que los maximizadores $\left(t_{[194]}=3.28, p \leq .05\right)$. También se encuentra una relación positiva entre la maximización y el pesar $(r=.393)$. Se discuten estos resultados en relación a los hallazgos obtenidos internacionalmente y a algunas características de la sociedad chilena.

Palabras clave: bienestar subjetivo, maximización, satisfacción, pesar.

\begin{abstract}
The purpose of this research was to evaluate the decision making using maximal and satisficing approaches and their effects on subjective well-being, according to its emotional component (happiness) and its cognitive dimension (satisfaction with the life). A total of 209 undergraduate students answered four questionnaires to asses maximization, satisfaction, regret and subjective well-being.

The result shows that there are significant differences between maximizers and satisficers as for the subjective well-being just in its cognitive component of vital satisfaction. The satisficers presents a better evaluation of their lives than the maximizers $\left(t\left[_{194]}=3.28, p \leq .05\right)\right.$ Finally a positive relation was found between maximizers and the tendency to express regret $(r=.393)$. These finds are discussed in based to internationally ones. Key words: subjetive well-being, maximizers, satisficers, regret.
\end{abstract}

\section{Introducción}

Chile es considerado un milagro económico con un ingreso per cápita de los más altos en la región y con tasas de crecimiento económico relativamente altas pero sus indicadores de bienestar subjetivo (B.S.) muestran que los chilenos tienen una satisfacción vital (S.V.) y económica baja, al igual que la salud, de modo que existen costos ocultos del crecimiento económico no reflejados en los indicadores de ingreso per cápita ni de desarrollo humano (Rojas, 2011), tal como se les mide habitualmente. La sociedad chilena fue diagnosticada en 1998 como una de 'malestar' (PNUD, 2010) caracterizada por una muy débil cohesión social, una muy alta desconfianza en las relaciones interpersonales, en dónde las personas experimentaban mucha incertidumbre respecto del futuro, de temor a no tener a quien recurrir en caso de necesidad, de no disponer de los recursos para afrontar enfermedades o problemas de su propia salud o la de sus hijos. Posteriormente y de modo convergente, estudiando la relación entre suicidio y Producto Interno Bruto (PIB) en Chile para los últimos 20 años, Moyano y Barría (2006) reportaron una correlación alta positiva y

Correspondencia: Emilio Moyano-Díaz, Facultad de Psicología, Universidad de Talca, Campus Lircay s/n. e.mail: emoyano@utalca.cl Agradecimientos: al Programa de Investigación Calidad de Vida y Ambientes Saludables (VAC600426) de la Facultad de Psicología, Universidad de Talca, Chile. 
significativa entre crecimiento del PIB y una tasa sostenida de aumento del suicidio en el período. Así, el conjunto de estos resultados dan apoyo a lo que ha sido denominado 'paradoja de la felicidad' (Easterlin, 1974, 1995; Bruni 2002, 2004), referida a que incrementos sustanciales en el ingreso no corresponden a incrementos equivalentes en niveles de felicidad.

Los signos referidos como de malestar en la sociedad chilena han tenido lugar en una sociedad en la cual su modelo de desarrollo fue cambiado mediante la fuerza (11/09/1973)-de orientado colectivistamente a otro orientado individualísticamente-, donde las personas son concebidas más como consumidores que como ciudadanos, y donde los jóvenes son alentados con insistencia desde las políticas económicas -y del sistema educativo- al emprendimiento como un valor central. La orientación pro emprendimiento enfatiza el valor de la capacidad de generar un negocio, ser su propio empleador, de ganar dinero, de maximizar. Realizar estas acciones y, más generalmente, las consecuencias de nuestro comportamiento, pueden llevarnos a experimentar emociones positivas o negativas y, entre estas, la felicidad ha estado ocupando un lugar importante en la investigación psicológica actual, especialmente desde el surgimiento de la psicología positiva con Seligman (2002) y trabajos posteriores (Haidt, 2006; Carr, 2004; Diener y Seligman, 2002; Moyano, 2010; Baucells y Sarin, 2012).

Entre las distintas líneas de investigación al respecto, un campo específico de interrogación es el relacionado con la clasificación de las personas según su modo de decidir y, por tanto, de actuar. Ha sido propuesto distinguir entre maximizadores y satisfacedores según su tendencia, respectivamente, a examinar y evaluar muchas opciones posibles antes de inclinarse por alguna o, simplemente, conformarse con la mejor entre las que aparecen primeramente disponibles, y dónde las consecuencias emocionales de proceder según una u otra tendencia serían diferentes (Schwartz et al., 2002). Según este autor último autor, la maximización en tanto atributo implica unidimensionalidad y bipolaridad, es decir se es satisfacedor o maximizador, pero no ambos.

De acuerdo a Schwartz et al. (2002) los satisfacedores son personas que típicamente no se detienen a analizar mucho entre las opciones posibles, y generalmente escogen lo que les parece suficientemente bueno, o una opción simplemente satisfactoria. Podríamos decir que tal vez operan con el refrán popular de que "lo óptimo es enemigo de lo bueno". Por el contrario, las del segundo tipo buscan escoger la mejor opción en orden a tener el mejor resultado, es decir, tenderán a invertir energía en analizar todas las alternativas posibles para poder estar seguros de lo que están escogiendo. La literatura muestra que tener una $\mathrm{u}$ otra tendencia al decidir trae efectos diferentes sobre el B.S. (Dar-Nimrod, Rawn, Lehman, y Schwartz, 2009; Purvis, Howell y Iyer, 2011).

Cuando los maximizadores buscan la 'mejor opción posible' frecuentemente realizan comparación social con otros, no sucediendo así con los satisfacedores, quienes al buscar sólo lo 'suficiente o bastantemente bueno' pueden juzgar o elegir en términos absolutos, viéndose menos afectados por la comparación social que los maximizadores (Schwartz, et. al., 2002). Se sabe que cuando no existen estándares objetivos que sirvan como criterio para opinar o decidir acerca de algo las personas tienden a evaluar sus propias opiniones, pareceres y decisiones mediante la comparación con las de los demás, preferentemente con personas pertenecientes a grupos sociales valorados por el sujeto. Ello permite confirmar o validar ciertas opiniones personales las que, entonces, adquirirán un carácter de estabilidad (Morales, 2002).

Lyubomirsky y Ross (1997) reportaron que las personas infelices se ven más afectadas por la comparación social ascendente, es decir, por la comparación con quienes obtienen un mejor resultado que el propio. Estudiantes que aparecían como infelices informaron sentirse más felices y seguros de sí mismos cuando habían recibido una evaluación pobre y habían sabido que otros habían recibido una evaluación peor, que cuando habían recibido una evaluación excelente y oído que otros tuvieron una evaluación aún mejor. En contraste, los estudiantes felices no mostraron este patrón al responder a las comparaciones sociales. Las personas maximizadoras al estar generalmente más predispuestas a la comparación social se sentirán entonces menos contentas o felices que las personas satisfacedoras (Pelusi, 2007).

De acuerdo a cuatro sucesivos estudios realizados por Schwartz et al. $(2000,2002)$ se concluye que existe una mayor probabilidad en maximizadores que en satisfacedores de realizar comparación social y, también, de que se vean más afectados por ella, sintiéndose menos felices y reportando mayor pesar con sus decisiones. En el primer estudio fueron creadas y validadas las Escalas de Maximización (EM) y la de Pesar (EP), las que miden respectivamente diferencias individuales en cuanto al deseo de maximizar o satisfacer, y la tendencia a experimentar pesar o malestar. En siete muestras se obtuvo consistentemente correlaciones negativas entre maximización y felicidad, optimismo, autoestima y satisfacción de vida, y correlaciones positivas entre la maximización y depresión, perfeccionismo y pesar (Schwartz et al. 2002). El segundo estudio mostró que los maximizadores se encontraron menos satisfechos que los satisfacedores al enfrentar decisiones de consumo y más 
proclives de asumir comparaciones sociales (Schwartz et al. 2002). En el tercer estudio de Schwartz et al. (2002) se informa que los maximizadores se encontraron afectados por la comparación social ascendente y, por último, en el cuarto estudio, fue reportado que los maximizadores son más propensos al arrepentimiento y manifiestan menos satisfacción con sus resultados en un juego de negocios (Schwartz et al. 2002).

A pesar de que distintas investigaciones muestran que los satisfacedores son en general más felices que los maximizadores, Schwartz no sostiene que haya que ser generalizadamente un satisfacedor en todos los dominios de la vida, indicando que existen decisiones en las que se debe ser un maximizador de todos modos (Lai, 2011), por ejemplo, al momento de elegir un pediatra para el hijo (Peterson, 2006).

En resumen, una persona maximizadora siempre va a querer la 'mejor u óptima opción', lo que involucra una exhaustiva búsqueda de posibilidades, y tendrá un sentimiento anticipado de pesar sobre la opción que tomará porque no logra conocer todas las posibilidades existentes. Incluso este sentimiento de pesar también se extiende sobre la opción elegida, al estar más propenso a realizar comparación social ascendente, lo que disminuye su bienestar e influye negativamente en su proceso de adaptación, al sentir que cada experiencia -de consumo por ejemplo- no le satisface completamente. Este aspecto no siempre ha sido considerado en algunas de las fórmulas para entender la felicidad (Haidt, 2006; Baucells y Sarin, 2012, por ejemplo), no obstante las elecciones entre pocas o muchas oportunidades en cada uno de los diferentes ámbitos de la vida parecen conducir a decisiones personales que causan efectos diferenciados en el bienestar y felicidad de las personas y, donde diferentes estudios, -desde Schwartz (2002) - han mostrado que los maximizadores experimentan más pesar y menos bienestar que los satisfacedores (Dar Nimrod, Rawn, Lehman y Schwartz, 2009; Purvis et al., 2011). Más específicamente, ha sido reportado que algunos rasgos de personalidad de la teoría de los cinco grandes como la extroversión, conciencia y neuroticismo (este último negativamente) están asociados a satisfacción vital (Fagley, 2012), y que algunos valores como la autodirección, y estimulación lo están a afecto positivo (Savig y Schwartz, 2000), y aún otros han agregado el universalismo (Roccas, Sagiv, Schwartz y Knafo, 2002). En otros se ha verificado que cuando se compara la contribución de los rasgos respecto de los valores, los primeros determinan más el bienestar subjetivo que los segundos (Haslam, Whelan y Bastian, 2009).
En el presente estudio se busca verificar que tan maximizadores o satisfacedores son un grupo de estudiantes universitarios chilenos, $\mathrm{y}$, también, identificar si una u otra tendencia decisional aparece o no asociada a su nivel de felicidad o pesar. Así, las preguntas que guían este trabajo son ¿Existe alguna tendencia de decisión dominante -maximización o satisfacción- en esta muestra de jóvenes universitarios?, ¿Cuáles es la relación entre tendencia decisional predominante -maximización o satisfacción-y la satisfacción vital y la felicidad como componentes del B.S.? Finalmente también resulta relevante responder ¿Cuáles son los eventuales efectos de dichas tendencias -maximización/ satisfacción- sobre el pesar?

En consecuencia, identificaremos la proporción de satisfacedores y maximizadores en la muestra de estudiantes universitarios estudiada, así como sus niveles de felicidad y de satisfacción vital y pesar, y la relación de éstas con sus tendencias decisionales dominantes. Siguiendo la literatura revisada (Schwartz, 2000, Schwartz, et al. 2002, Drakopoulos, 2008, Lai, 2010), se espera encontrar más maximizadores que satisfacedores, y que éstos últimos presenten más B.S. y menos pesar que los maximizadores.

\section{Método}

\section{Participantes}

Se extrajo una muestra de 211 estudiantes universitarios, usando un muestreo por conveniencia y mediante colaboración voluntaria. De los 211 participantes, cuyas edades fluctuaron entre 18 y 34 años, con promedio de 21.8 años y una moda de 20 años, se obtuvo 209 encuestas correctamente completadas. Un $67 \%$ corresponde a mujeres y un $33 \%$ a hombres lo que representa muy bien la proporción real de estudiantes de psicología (mujeres $72.8 \%$ y hombres $27.2 \%$ ). Participaron estudiantes pertenecientes a todos los niveles del plan de estudios, aunque el mayor porcentaje correspondió a tercer año (28\%) y el menor a primer año (8.6\%). Un $57 \%$ de los participantes eran solteros, $41 \%$ estaban en una relación con algún grado de compromiso, los casados correspondían al $1.4 \%$ y los separados $0.5 \%$.

\section{Instrumentos}

Se utilizó 4 instrumentos: las escalas de Maximización (EM) y de Pesar (EP) (Schwartz et al., 2002), la escala de Felicidad subjetiva (EFS) (Lyubomirsky y Lepper, 1999), la escala de Satisfacción Vital (ESV) (Diener y Emmons, 1985). 
Escala de Maximización (EM): creada por Schwartz et al. (2002) para evaluar la tendencia a la maximización y a la satisfacción a partir de siete muestras -cuatro de estudiantes universitarios de psicología y tres de adultos de población general- con 1747 participantes estadounidenses. Está compuesta por 13 afirmaciones, en formato de respuesta likert de 7 puntos, que va de completamente en desacuerdo a completamente de acuerdo. La consistencia interna original es de 0.71 y para esta investigación resultó de 0.69 .

Escala de Pesar (EP): creada por Schwartz et al. (2002) para evaluar la tendencia a experimentar pesar o malestar, está compuesta por 5 ítems tipo likert de 7 puntos, donde 1 es completamente en desacuerdo y 7 completamente de acuerdo. La consistencia interna original (Schwartz et al., 2002) es de 0.67 , y de 0.71 para esta investigación.

Escala de Felicidad Subjetiva (EFS): de Lyubomirsky y Lepper (1999) compuesta por cuatro ítems, en formato de respuesta likert de 1 a 7 puntos. Se utiliza la versión chilena realizada por Moyano y Ramos (2007) que posee una alfa de Cronbach de 0.79 concordante con la validación realizada por Vera, Célis y Córdova (2011).

Escala de Satisfacción Vital (ESV): creada por Diener y Emmons (1985), consiste en 5 ítems tipo likert de 7 puntos $(1=$ muy en desacuerdo y $7=$ muy de acuerdo $)$ cuyo puntaje total va de 5 y a 35 puntos. Tanto en su versión original anglo norteamericana como en la versión castellana chilena de Moyano y Ramos (2007) la consistencia interna de este instrumento es de 0.87 .

\section{Procedimiento}

Se obtuvo la autorización de las autoridades académicas de la Escuela de Psicología de la Universidad y el consentimiento informado de parte de quienes aceptaron participar de modo que los cuestionarios fueron aplicados en los lugares habituales frecuentados por los estudiantes (sala de estar, aulas, en el recinto de la fotocopiadora, laboratorios de psicoinformática, etc.). Se realizó análisis estadísticos descriptivos respecto de maximización, pesar, felicidad y satisfacción vital. Posteriormente fue realizada una comparación de medias mediante la prueba t para muestras independientes para satisfacedores y maximizadores en relación al pesar y al B.S. Finalmente, fue estimado el tamaño del efecto de estas diferencias usando la diferencia media tipificada o $d$ de Cohen y se realizó correlaciones de Pearson para establecer el grado de asociación entre las variables. Todos los análisis se realizaron usando el programa SPSS 15.0.

\section{Resultados}

Descripción general: maximizadores, satisfacedores, felicidad y satisfacción vital.

En esta muestra de universitarios hay una mayor cantidad de maximizadores que de satisfacedores $(52.6 \% ; f=110$, versus $41.1 \% ; f=86$, respectivamente). Fueron considerados "neutros" quienes no poseen una tendencia marcada en sus estilos de decisión y, por lo tanto, no pertenecen a ninguno de los grupos de interés. Un maximizador puntúa entre 1 y 3.9 puntos y un satisfacedor entre 4.1 y 7 puntos, de modo que se considerará como neutros(as) a quienes puntúan 4 . Estos constituyen sólo un $6.2 \%$ del total $(f=13)$,

Se observa que los participantes de esta muestra son relativamente felices $(M=5.17$ sobre $7 ; D T=1.01)$, de modo que perciben su vida como buena, experimentando más frecuentemente emociones positivas que negativas. En cuanto a su satisfacción vital se observa una valoración cognitiva positiva de la propia vida como buena $(M=$ 5.1 sobre 7, $D T=1.06$ ). En síntesis, se establece que los participantes en general presentan un nivel razonable de BS que podemos considerar como correspondiente a un punto intermedio entre las categorías de 'más que regular' $\mathrm{y}$ 'buen nivel' de BS.

Felicidad y satisfacción vital y pesar en maximizadores y satisfacedores.

Los resultados de la prueba $\mathrm{T}$ para muestras independientes indican que los satisfacedores poseen una media ligeramente superior a los maximizadores en felicidad, sin embargo, esta diferencia no resulta significativa ( $p=$ .164) de modo que no se confirma la primera hipótesis de estudio. Por otro lado, sí se confirma la tercera hipótesis relativa al componente cognitivo del B.S. -la satisfacción vital- ya que se obtiene diferencias entre maximizadores y satisfacedores siendo estos últimos los que puntúan significativamente más alto en la escala, aunque con un efecto pequeño (Tabla 1$)(p \leq .05 ; d=.470)$. Por lo tanto, se puede decir que los satisfacedores tienen mayor bienestar que los maximizadores sólo en cuanto al componente cognitivo del BS -la satisfacción vital-, y no en el componente afectivo-felicidad-para el cual no se encuentra diferencias significativas.

Al analizar la variable pesar y, del mismo modo que lo reportado por Schwartz et al. (2002), se observa que los 
maximizadores puntúan significativamente más alto que los satisfacedores $(p \leq .05)$. Esta diferencia es confirmada a partir de las diferencias mínimas tipificadas, indicando un tamaño de efecto mediano $(d=-.770)$. Para el caso de la felicidad (EFS) y la satisfacción vital (ESV) el tamaño del efecto de las medias es pequeño $(d s \leq .50)$.

\section{Relaciones entre BS, maximización y pesar.}

Respecto del grado de asociación entre las variables (tabla 2), puede ser observado que las que presentan una relación significativa con la maximización son la satisfacción vital y el pesar. Existe una correlación negativa (baja), entre maximización y satisfacción vital $\left(r_{[209]}=-.179 ; p=.009\right)$, y una positiva -y moderada- entre maximización y la Escala de Pesar $\left(r_{[209]}=.393 ; p=.001\right)$. Esto significa que a medida que aumenta la maximización o mientras más maximizador se es, menor nivel de satisfacción vital se tendrá y mayor pesar será experimentado. Así, según esto último, la tercera hipótesis es confirmada.

Como era esperable, se observa una relación significativa y positiva entre satisfacción vital y felicidad $\left(r_{[209]}=.583\right.$; $p=.001)$. Adicionalmente, se observa una correlación moderada, significativa y negativa entre felicidad y pesar, de modo que a mayor puntaje en la escala de pesar menor felicidad reportan las personas $\left(r_{[209]}=-.211 ; p=.002\right)$.

\section{Discusión}

En el presente estudio se identifica la relación existente entre los componentes del bienestar subjetivo -felicidad y satisfacción vital- con la tendencias a decidir siguiendo estilo maximizador o satisfactor, y un primer resultado general es que el nivel de B.S. de la muestra de estudiantes de psicología es satisfactorio. En segundo lugar, fue observado que existe una mayor cantidad de maximizadores que satisfacedores en esta muestra de estudiantes universitarios. Aún cuando no contamos con información histórica o longitudinal específica al respecto, tal vez ello puede ser interpretado como un efecto persuasivo del discurso hacia el emprendimiento y desarrollo de las capacidades de negocio entre la población joven universitaria, característico del Chile de los últimos años.

Tabla 1. Comparación de medias de maximizadores y satisfacedores respecto de EFS, ESV y Escala de Pesar ( $n=86$ y 110 para satisfacedor y maximizador respectivamente).

\begin{tabular}{lcccccc}
\hline & Categorización & Media & Desviación típ. & $\begin{array}{c}\text { Error típ. de la } \\
\text { media }\end{array}$ & Sig. & $d$ \\
\hline Escala de Pesar & Satisfacedor & 3.684 & 0.903 & 0.097 & .000 & -.770 \\
& Maximizador & 4.420 & 0.988 & 0.094 & & .200 \\
EFS & Satisfacedor & 5.323 & 0.989 & 0.107 & .164 & .009 \\
& Maximizador & 5.121 & 1.019 & 0.097 & .470 \\
ESV & Satisfacedor & 5.405 & 0.884 & 0.108 & .001 & \\
\end{tabular}

Tabla 2. Matriz de correlaciones de maximización, ESV, EFS y pesar $(n=209)$.

\begin{tabular}{lccccc}
\hline & & $\begin{array}{c}\text { Escala de } \\
\text { Maximización }\end{array}$ & ESV & EFS & $\begin{array}{c}\text { Escala de } \\
\text { Pesar }\end{array}$ \\
\hline Escala de Maximización & Correlación de Pearson & 1 & $-0.179^{* *}$ & -.115 & $.393^{* *}$ \\
& Sig. (bilateral) & & 0.009 & .098 & .000 \\
ESV & Correlación de Pearson & & 1 & $.583^{* *}$ & $.302^{* *}$ \\
& Sig. (bilateral) & & .000 & .000 \\
EFS & Correlación de Pearson & & 1 & $.211^{* *}$ \\
Escala de Pesar & Sig. (bilateral) & & & .002 \\
& Correlación de Pearson & & & 1 \\
\hline
\end{tabular}

Nota. ${ }^{* *}$ La correlación es significativa al nivel 0.01 (bilateral). 
Respecto a la hipótesis que establece que los satisfacedores tienen mayores niveles de bienestar subjetivo que los maximizadores, se confirma en este caso sólo para el componente cognitivo del B.S., es decir, sobre la satisfacción vital. Los satisfacedores manifiestan mayor valoración de tipo cognitivo sobre su propia vida, existiendo por ello una aproximación más cercana entre sus expectativas y sus logros. Esta relación positiva entre satisfacción vital y tendencia decisional satisfacedora sería semejante a la encontrada en el estudio de Schwartz et al. (2002) en donde los satisfacedores presentaron mayor satisfacción de vida $-\mathrm{y}$ también mayor felicidad, optimismo, autoestima-, y significativamente menor pesar y depresión que los maximizadores. De acuerdo a esto, la relación entre los niveles de satisfacción vital y la tendencia decisional satisfacedora de la muestra parece congruente con pertenecer a sociedad donde ciertas metas y objetivos se encuentran predeterminados, como por ejemplo, terminar la educación media e ingresar a la universidad, formar una familia o conseguir un trabajo que genere los mayores ingresos y niveles de realización personal posibles. Una meta principal característica del período etáreo en que se encuentran los participantes del estudio corresponde a aprobar sus estudios y terminar su carrera profesional, y dado que esto está actualmente consiguiéndose existe entonces una relación congruente entre logros y expectativas y, por ende, parece probable una valoración positiva respecto de cómo va transcurriendo la vida.

Los maximizadores por su parte también -como en Schwartz et al. (2002)- presentan un menor nivel de satisfacción vital que los satisfacedores. Aparentemente tienen mayores expectativas que los satisfacedores, y sin embargo se van sintiendo menos satisfechos con los logros obtenidos. Tal vez la explicación a ello sea su propensión a la comparación social ascendente, lo que les provocaría mayor probabilidad de experimentar pesar, lo que redunda en un menor nivel de satisfacción vital, lo cual podría ser sometido a verificación en estudios futuros.

En relación a la segunda hipótesis, referida a que los índices de felicidad de los satisfacedores serían mayores que los de los maximizadores, si bien los promedios obtenidos están en la dirección esperada, la diferencia obtenida no fue suficiente para alcanzar significación estadística, difiriendo así de los resultados obtenidos por Schwartz et al. (2002). Hay que señalar que los niveles de felicidad de ambos grupos son aceptables, y el hecho que no se diferencien significativamente entre si tal vez se deba a que los estudiantes comparten muchas características relevantes, tal como vivir mayoritariamente en ambientes 'protegidos', es decir, con opciones limitadas dada su situación de dependencia económica o de limitados recursos económicos -incluso para los que eventualmente trabajan-, en donde se produce un autocontrol -no necesariamente 'voluntario' u opcional-de sus actividades, comportamientos, y experiencias de consumo. Esto podría explicar que no aparezcan diferencias tan marcadas entre ellos no obstante sus diferencias de tendencias decisionales. Independientemente de la edad, los niveles de felicidad encontrados en la muestra en estudio no difieren de los encontrados en otros estudios como por ejemplo, el de Moyano y Ramos (2007), o el de Vera, Celis y Córdova (2011) que incluyeron diferentes muestras de población chilena, obteniendo resultados similares en cuanto a felicidad.

En términos generales, lo anterior es convergente con los datos que ubican a Chile, en comparación con el resto de los países de Latinoamérica, en el segundo nivel inferior o más bajo en felicidad (CIMA Group, 2006) no obstante tratarse del país con mayor ingreso per cápita de los países considerados en ese estudio. Por otro lado, al estar en una situación de bienestar económico creciente, dentro de un modelo capitalista, la mayoría de las personas ven satisfechas sus necesidades básicas, y así también van manifestando mayor nivel de satisfacción y felicidad que se traducen, en este período de tiempo, como aceptables. Chile tiene un PIB per cápita de US16.172 (Fondo Monetario Internacional, FMI, 2011) y, según Layard (2005) esta cifra es el límite superior a partir del cual la felicidad no aumenta más y más bien tiende a disminuir. La relación entre ingreso y felicidad parece ser curvilineal como lo han señalado Veenhoven (1991) y Lane (2000). Así, resulta razonable pronosticar que la felicidad en Chile no incrementará sino que permanecerá -en el mejor de los casos- en su nivel actual.

Esto además se complementa con la valoración que los chilenos otorgan al dinero, ya que entre los países de Latinoamérica Chile es el país donde más importancia se le da a éste, y simultáneamente es el aspecto de la vida que menos satisfechos tiene a los ciudadanos (Tironi, 2006; Herrera, Estrada y Denegri, 2011). Esto concuerda con la formulación de Seligman (2002) de que las personas que valoran el dinero más que otros aspectos de la vida están menos satisfechas con sus ingresos y con su vida en general. Si los chilenos tienen la tendencia a valorar más el dinero que otros aspectos de la vida, ello es un factor que también contribuye el malestar o pesar que parece vivirse en amplios sectores de la sociedad hoy. Posiblemente el crecimiento del ingreso per cápita alcanzado en el país junto con la gran importancia que los chilenos le asignan al dinero, sean algunos de los factores que influyeron aquí para que no se haya comprobado la hipótesis de encontrar niveles de felicidad superiores en satisfacedores que en maximizadores, como 
Schwartz et al. (2002) lo ha encontrado en una muestra de estadounidenses cuyo PIB nacional per cápita es superior al chileno (US 37.600 para el año 2002; FMI 2011), y una cultura distinta.

Finalmente, la última hipótesis de este estudio, referida a que los maximizadores experimentarían más pesar que los satisfacedores se confirma, ya que fue encontrada una relación significativa y positiva, como en Schwartz et al. (2002). Según sabemos, los maximizadores al buscar el mejor resultado están más afectados por la comparación social, debido a que la valoración de su resultado sólo se ve en relación a sus iguales (o ‘superiores'). Por lo tanto su atención al actuar -como energía psíquica-y determinar lo que aparecerá en su conciencia (Csikszentmilhayi, 2004), está llevando normalmente información de su desempeño y de las opciones elegidas en relación a los otros, especialmente comparación social ascendente, la que es característico de las personas infelices. Ello puede dificultar su proceso de adaptación en los diferentes ámbitos de la vida (Lyubomirsky y Ross, 1997).

Es importante también considerar que en el mundo moderno las opciones en todo ámbito (salvo tal vez el político) -y especialmente en la oferta de productos de consumo- han ido en aumento, lo que deja a las personas como artífices o constructores de sus propias realidades y destinos, en un escenario que presenta múltiples oportunidades, y dónde una de las pocas certezas es que es imposible evaluar todas las opciones posibles. Por cierto, esto tiene límites, especialmente en sociedades altamente desiguales como la chilena (coeficiente de Gini 0.56) donde el campo de lo elegible es extraordinariamente más amplio para quienes pertenecen al estrecho segmento de la riqueza. Siguiendo los resultados de la literatura revisada y de lo encontrado en el presente estudio, la incrementada oferta de alternativas genera búsqueda o análisis de las mejores alternativas, fomentándose una tendencia decisional orientada hacia la maximización y, disminuyendo así el bienestar subjetivo. Por ello, profundizar el estudio de este tema resulta de vital importancia a la hora de buscar modos de mitigar el pesar o malestar y la infelicidad, especialmente en una sociedad como la chilena con altos niveles de depresión y de incrementada tasa de suicidio (Moyano y Barría, 2006) y caracterizable como una en la cual la paradoja de la felicidad resulta paradigmática (Drakopoulos, 2008). Se puede derivar de este tipo de resultados formas de reeducación de las personas respecto de sus tendencias decisionales en sus proyectos de vida, promoviendo una educación para la felicidad. Los componentes de una 'buena vida' o el 'buen vivir' (Rojas, 2009) pueden ser parte de las acciones de prevención que los psicólogos realicen para mejorar la calidad de vida de las personas, y debieran ser considerados en política pública educacional y sanitaria.

Resultaría interesante replicar este estudio en diferentes muestras, que incluyan diversos grupos etáreos, niveles socioeconómicos y ocupación de los participantes, porque la literatura en torno a la felicidad muestra algunas divergencias en relación a las variables mencionadas (Diab, Gillespie y Highhouse, 2008; Kuhnle y Sinclair, 2011; Bin, Turner, Betz y Nygren, 2011). Identificar si la maximización varía en estos diferentes grupos, así como conocer su grado en personas de distinto nivel educacional, religión, ideología o estilo de vida, ayuda a orientar y focalizar política pública. Por otra parte, otra interrogante posible para próximos estudios, es identificar que rasgos de personalidad se encuentran a la base de personas maximizadoras o satisfacedoras o si es que estas disposiciones -maximizar y satisfacer- van más allá o constituyen per se, un estilo de personalidad.

\section{Referencias}

Baucells, M., y Sarin, R. (2012). Engineering happiness. A new approach for building a joyful life. University California Press.

Bin, H., Turner, B., Betz, N., y Nygren, T. (2011).Studies of the dimensionality, correlates, and meaning of measures of the maximizing tendency. Judgment and Decision Making, 6, 565-579.

Bruni, L. (2002). A history of happiness in economics. Paper presented to the European Society for the History of Economic Thought Conference, Crete.

Bruni, L. (2004). The "happiness transformation problem" in the Cambridge tradition. European Journal of the History of Economic Thought, $11,431-451$.

Carr, A. (2004). Psicología positiva: la ciencia de la felicidad. Barcelona, España: Paidos, Psicología, Psiquiatría y Psicoterapia.

CIMA Group. (2006). Estudio Sobre la Felicidad de los Chilenos (Informe Preliminar). Santiago, Chile: Autor, CIMA Group, Unidad de Información y Análisis de Mercados.

Csikszentmihalyi, M. (2004). Fluir. Barcelona: Kairos.

Dar-Nimrod, I., Rawn, R., Lehman, D., y Schwartz, B. (2009). The Maximization Paradox: The costs of seeking alternatives. Personality and Individual Differences 46, 631-635.

Diab, D., Gillespie, M., y Highhouse, S. (2008). Are maximizers really unhappy? The measurement of maximizing tendency. Judgment and Decision Making, 3, 364-370

Diener, E., y Emmons, R. (1985). The independence of positive and negative affect. Journal of Personality and Social Psychology, 47, 1105-1117.

Diener, E., y Seligman, M. (2002). Very happy people. Psychological Science, 13, 81-84.

Drakopoulos, S. A. (2008). The paradox of happiness: towards an alternative explanation. J. Happiness Studies, 9, 303-315.

Easterlin, R. A. (1974). Does economic growth improve the human lot?. In P. A. David and M. W. Reder (Eds). Nations and Households in Economic Growth: Essays in Honor of Moses Abramovitz. New York: Academic Press, Inc.

Easterlin, R. A. (1995). Will raising the incomes of all increase the happiness of all?. Journal of Economic Behavior and Organization, 27, 35-48. 
Fagley, N. S. (2012). Appreciation uniquely predicts life satisfaction above demographics, the Big 5 personality factors, and gratitude. Personality and Individual Differences, 53, 59-63.

Fondo Monetario Internacional (2011). World Economic Outlook Database. Recuperado desde http://www.imf.org/external/pubs/ft/weo/2011/02/ weodata/index.aspx

Haidt, J. (2006). La hipótesis de la felicidad. España: Gedisa.

Haslam, N., Whelan, J., y Bastian, R. (2009). Big Five traits mediate associations between values and subjective well-being. Personality and Individual Differences. 46, 40-42.

Herrera, M. G., Estrada, C. A., y Denegri, M. (2011). Economic literacy, consumer behavior, attitude towars debt and its relation to psychological well-being in public employees of the city of Punta Arenas. Magallania, 39, 83-91.

Kuhnle, C., y Sinclair, M. (2011). Decision mode as an antecedent of flow, motivational interference, and regret. Learning and Individual Differences 21, 239-243.

Lai, L. (2010). Maximizing without difficulty: A modified maximizing scale and its correlates. Judgment and Decision Making, 5, 164-175.

Lai, L. (2011). Maximizing and customer loyalty: Are maximizers less loyal? Judgment and Decision Making, 6, 307-313.

Lane, R. (2000). The loss if happiness in the market democracies. Yale: Yale University Press.

Layard, R. (2005). Happiness: Lesson from a new science. Estados Unidos: Hardcover

Lyubomirsky, S., y Ross, L. (1997). Hedonic consequences of social comparison: A contrast of happy and unhappy people. Journal of Personality and Social Psychology, 73, 1141-1157.

Lyubomirsky, S., y Lepper, H. (1999). A Measure of subjective happiness: Preliminary reliability and construct validation. Social Indicators Research, 46, 137-156.

Morales, J. F. (2002). La emigración como forma de exclusión social. En Morales, F., Páez, D., Kornblit, A. L. y Asún, D. (coords.), Psicología Social (pp. 127-137). Buenos Aires: Prentice Hall.

Moyano, E., y Barría, R. (2006). Suicidio y Producto Interno Bruto (BIP) en Chile: hacia un modelo predictivo. Revista Latinoamericana de Psicología, 38, 343-359.

Moyano, E., y Ramos, N. (2007). Bienestar subjetivo: midiendo satisfacción vital, felicidad y salud en población chilena de la región del Maule. Revista Universum, 2, 1-17.
Moyano, E. (2010). Calidad de vida y psicología en el Bicentenario de Chile. Editorial Marmor: Talca, Chile.

Pelusi, N. (2007). When to choose is to lose. Psychology Today, 40, 69-71.

Peterson, C. (2006). A primer in Positive Psychology. Oxford: Oxford University Press.

PNUD (2010). Informe sobre el desarrollo humano 2010. Recuperado desde Human development reports: http://hdr.undp.org/en/media/ HDR_2010_ES_Tables_reprint.pdf

Purvis, A., Howell, R., y Iyer, R. (2011). Exploring the role of personality in the relationship between maximization and well-being. Personality and Individual Differences 50, 370-375.

Roccas, S., Sagiv, L., Schwartz, S. H., y Knafo, A. (2002). The Big Five personality factors and personal values. Personality and Social Psychology Bulletin, 28, 789-801.

Rojas, M. (2009). Monetary valuation of illnesses in Costa Rica: a subjective well-being approach. Revista Panamericana de Salud Pública, 26, 255-265.

Rojas, M. (2011). Más allá del ingreso: progreso y bienestar subjetivo. En: M. Rojas (Coord.), La medición del progreso y del bienestar. Propuestas desde América Latina (pp. 29-39). Foro Consultivo Científico y Tecnológico, AC México.

Savig, L., y Schwartz, S. H. (2000). Values priorities and subjective wellbeing: Direct relations and congruity effects. European Journal of Social Psychology, 30, 177-198.

Schwartz, B. (2000). Self-Determination: The tyranny of fredom. American Psychologist, 55, 79-88.

Schwartz, B., Monterosso, J., Lyubomirsky, S., White, K., y Lehman, D. (2002). Maximizing Versus Satisficing: Happiness Is a Matter of Choise. Journal of Personality and Social Psychology, 83, 1178-1197.

Seligman, M. (2002). La auténtica felicidad. Barcelona: Byblos.

Tironi, E. (2006). Crónica de viaje: Chile y la ruta a la felicidad. Santiago: El Mercurio Aguilar.

Veenhoven, R. (1991). Is happiness relative? Social Indicators Research 24, 1-34.

Vera-Vllarroel, P., Celis-Atenas, K., y Córdova, N. (2011). Evaluación de la Felicidad: Análisis Psicométrico de la Escala de Felicidad Subjetiva en Población Chilena. Terapia Psicológica, 29, 127-133. 